\title{
Response of a uniformly accelerated detector to massless Rarita-Schwinger fields in vacuum
}

\author{
Qinglin $\mathrm{Li}^{1}$, Hongwei $\mathrm{Yu}^{1,2, *, \dagger}$ and Wenting Zhou ${ }^{2}$ \\ 1 Department of Physics and Key Laboratory of Low Dimensional \\ Quantum Structures and Quantum Control of Ministry of Education, \\ Hunan Normal University, Changsha, Hunan 410081, China \\ ${ }^{2}$ Center for Nonlinear Science and Department of Physics, \\ Ningbo University, Ningbo, Zhejiang 315211, China
}

\begin{abstract}
We study the response of a uniformly accelerated detector modeled by a two-level atom nonlinearly coupled to vacuum massless Rarita-Schwinger fields. We first generalize the formalism developed by Dalibard, Dupont-Roc, and Cohen-Tannoudji in the linear coupling case, and we then calculate the mean rate of change of the atomic energy of the accelerated atom. Our result shows that a uniformly accelerated atom in its ground state interacting with vacuum RaritaSchwinger field fluctuations would spontaneously transition to an excited state and the unique feature in contrast to the case of the atom coupled to the scalar, electromagnetic and Dirac fields is the appearance of terms in the excitation rate which are proportional to the sixth and eighth powers of acceleration.

PACS numbers:
\end{abstract}

\footnotetext{
* Corresponding author

† email: hwyu@hunnu.edu.cn
} 


\section{INTRODUCTION}

Unruh discovered in1976, by examining the response of a so-called Unruh-DeWitt particle detector to massless scalar fields, that for a uniformly accelerated observer, the Minkowski vacuum is seen to be equivalent to a thermal bath of Rindler particles at a temperature $T_{U}=a / 2 \pi$ [1], where $a$ is the observer's proper acceleration. Since then, the Unruh effect has attracted a great deal of attention both for the crucial role it has played in our understanding that the particle content of a quantum field theory is observer dependent and for its close relationship to the Hawking radiation from black holes (see Refs. [2 4] for reviews for extensive works on the Unruh effect and its applications). Recently, the Unruh effect has been studied from the perspective of a two-level atom interacting with vacuum quantum fields, such as scalar [5-7], electromagnetic [8-13] and Dirac fields [14], in the formalism developed by Dalibard, Dupont-Roc, and Cohen-Tannoudji (DDC) [15, 16], where the atom's excitation is distinctively attributed to the contributions that result from fluctuations in the vacuum, and those that are due to the disturbance of the quantum field caused by the atom-field coupling. These studies show that if the atom is accelerated, then the delicate balance between vacuum fluctuations and radiation reaction that ensures the stability of the ground-state inertial atoms in vacuum is altered, thereby making transitions to excited states for the ground-state atoms possible even in vacuum. This result is not only consistent with the Unruh effect, but also provides a physically appealing interpretation of it, since it gives a transparent illustration for why an accelerated detector clicks.

In this paper, we plan to go a step further to make the spectrum of research more complete, that is, we will extend the studies on the Unruh effect to particle detectors coupled to quantum fields of higher spin. In particular, we will examine the spontaneous excitation of an accelerated detector modeled by a two-level atom in interaction with vacuum spin 3/2 fields, i.e., Rarita-Swhinger fields [17], using the DDC formalism that makes use of a well-defined separation of detector excitations into vacuum fluctuations and radiation reaction. Our interest in the issue also lies in the role Rarita-Schwinger fields play in supersymmetry. As it is well-known, supersymmetry is a relativistic symmetry between bosons and fermions [18 20]. It unites in a single supermultiplet particles with different intrinsic spins differing by units of one half so that fermions become superpartners of bosons and vice versa. If it is correct, supersymmetry implies that every known elementary particle must have a superpartner. So, the graviton of spin 2 supposed to mediate gravitation, one of the four fundamental interactions in nature, has a superpartner called the gravitino which is described a spin- $\frac{3}{2}$ field, i.e., the Rarita-Schwinger field. We plan to calculate the spontaneous excitation rate of a uniformly accelerated two-level atom in interaction with vacuum Rarita-Schwinger fields using the DDC formalism.

The paper is organized as follows. In Sec. II, we generalize the DDC formalism to RaritaSchwinger fields where the coupling between an atom and the field is nonlinear and then in Sec. III, we use the generalized DDC formalism to calculate the spontaneous excitation rate of uniformly accelerated atoms interacting with fluctuating Rarita-Schwinger fields in 
vacuum. We conclude in Sec. IV. Natural units in which $\hbar=c=1$ and metric signature $(+,-,-,-)$ will be used throughout the paper.

\section{THE GENERAL FORMALISM}

The system we shall consider consists of a two-level atom and a bath of vacuum fluctuating massless Rarita-Schwinger fields in four dimensional Minkowski spacetime. The two states of the atom, i.e., the ground and excited states, are denoted by $|-\rangle,|+\rangle$, respectively, with energies being $-\frac{1}{2} \omega_{0}$ and $+\frac{1}{2} \omega_{0}$. The coordinates of the atom associated with an inertial reference are denoted by $x^{\mu}=\left(x^{0}, \vec{x}\right)=(t, x, y, z)$. The atom is assumed to be on a stationary trajectory $x(\tau)=(t(\tau), \vec{x}(\tau))$ where $\tau$ indicates the proper time. The evolution of the atom in proper time $\tau$ is controlled by the following Hamiltonian [21],

$$
H_{A}(\tau)=\omega_{0} R_{3}(\tau)
$$

where $R_{3}(0)=\frac{1}{2}|+\rangle\left\langle+\left|-\frac{1}{2}\right|-\right\rangle\langle-|$. The Lagrangian of the massless Rarita-Schwinger field $\psi_{\rho}(x)$ which the atom is assumed to be coupled to is given by [22]

$$
\mathcal{L}=-\epsilon^{\lambda \rho \mu \nu} \bar{\psi}_{\lambda} \gamma_{5} \gamma_{\mu} \partial_{\nu} \psi_{\rho}
$$

where

$$
\gamma^{0}=\left(\begin{array}{cc}
I & 0 \\
0 & -I
\end{array}\right), \quad \gamma^{i}=\left(\begin{array}{cc}
0 & \sigma_{i} \\
-\sigma_{i} & 0
\end{array}\right)
$$

with $\sigma$ being the Pauli matrices, $\gamma_{5}=i \gamma^{0} \gamma^{1} \gamma^{2} \gamma^{3}, \epsilon^{0123}=1$, and $\bar{\psi}_{\lambda}=\psi_{\lambda}^{\dagger} \gamma^{0}$. The $\gamma$ matrices satisfy the algebra: $\left\{\gamma^{\mu}, \gamma^{\nu}\right\}=2 g^{\mu \nu}$, where $\{$,$\} stands for the anticommutator.$ The Lagrangian (2) leads to the following Euler-Lagrange equation of motion

$$
i\left[g^{\lambda \rho} \not \partial-\gamma^{\lambda} \partial^{\rho}-\gamma^{\rho} \partial^{\lambda}+\gamma^{\lambda} \not \partial \gamma^{\rho}\right] \psi_{\rho}=0
$$

where $\not \partial=\gamma^{\alpha} \partial_{\alpha}$. This equation is invariant under chiral rotations as well as fermionic gauge transformations $\psi_{\rho} \rightarrow \psi_{\rho}+\partial_{\rho} \phi$, where $\phi$ is an arbitrary spinor field. To fix the gauge, we impose the following conditions [23]

$$
\gamma^{i} \psi_{i}=0, \quad \psi_{0}=0, \quad \partial^{i} \psi_{i}=0
$$

Then the original Euler-Lagrange equation becomes the Dirac-like equation of motion, $i \not \partial \psi_{\mu}=0$. Consequently, the Rarita-Schwinger field has a general plane-wave expansion [23],

$$
\psi_{\mu}(x)=\sum_{s= \pm \frac{3}{2}} \int \frac{d^{3} \vec{p}}{\sqrt{(2 \pi)^{3} 2 p_{0}}}\left[c(\vec{p}, s, t) u_{\mu}(\vec{p}, s) e^{-i p \cdot x}+d^{*}(\vec{p}, s, t) v_{\mu}(\vec{p}, s) e^{i p \cdot x}\right] .
$$


Here, the mode function $u_{\mu}(\vec{p}, s)$ is given by

$$
u_{\mu}\left(\vec{p}, \pm \frac{3}{2}\right)=\mathcal{D}_{3}(\phi) \mathcal{D}_{2}(\theta) u_{\mu}\left(p_{0}, \pm \frac{3}{2}\right)
$$

with

$$
u_{\mu}\left(p_{0}, \pm \frac{3}{2}\right)=\epsilon_{\mu}\left(p_{0}, \pm\right) u\left(p_{0}, \pm\right)
$$

where the polarization vectors, $\epsilon_{\mu}\left(p_{0}, \pm\right)$, and the Dirac spinors in the direction $p^{\mu}=$ $\left(p_{0}, 0,0, p_{0}\right)\left(p_{0}>0\right), u\left(p_{0}, \pm\right)$, are given respectively by

$$
\epsilon_{\mu}\left(p_{0}, \pm\right)=\mp \sqrt{\frac{1}{2}}(0,1, \pm i, 0)
$$

and

$$
u\left(p_{0}, \pm\right)=\sqrt{p_{0}}\left(\begin{array}{c}
\varphi_{ \pm} \\
\pm \varphi_{ \pm}
\end{array}\right) \text {with } \varphi_{+}=\left(\begin{array}{l}
1 \\
0
\end{array}\right), \varphi_{-}=\left(\begin{array}{l}
0 \\
1
\end{array}\right) .
$$

$\mathcal{D}_{3}(\phi) \mathcal{D}_{2}(\theta)$ is the rotation of $u_{\mu}\left(\vec{p}, \pm \frac{3}{2}\right)$ from the direction $p^{\mu}=\left(p_{0}, 0,0, p_{0}\right)$ to a generic direction $p^{\mu}=\left(p_{0}, p_{0} \sin \theta \cos \phi, p_{0} \sin \theta \sin \phi, p_{0} \cos \theta\right)=\left(p_{0}, \vec{p}\right)$. The mode function $v_{\mu}(\vec{p}, s)$ can be obtained by the relation $v_{\mu}(\vec{p}, s)=C \bar{u}_{\mu}^{T}(\vec{p}, s)$, where $C=i \gamma^{2} \gamma^{0}$ and $\bar{u}_{\mu}(\vec{p}, s)=$ $u_{\mu}^{\dagger}(\vec{p}, s) \gamma^{0}$. These mode functions are normalized such that

$$
\bar{u}^{\mu}\left(\vec{p}, s^{\prime}\right) \gamma^{\nu} u_{\mu}(\vec{p}, s)=\bar{v}^{\mu}\left(\vec{p}, s^{\prime}\right) \gamma^{\nu} v_{\mu}(\vec{p}, s)=-\delta_{s s^{\prime}} 2 p^{\nu}
$$

and

$$
\bar{u}^{\mu}\left(\vec{p}, s^{\prime}\right) \gamma^{0} v_{\mu}(\vec{p}, s)=0
$$

Here the anti-commutation relations of $c(\vec{p}, s)$ and $d^{*}(\vec{p}, s)$ as fermion creators and annihilators are

$$
\begin{array}{r}
\left\{c(\vec{p}, s), c^{*}\left(\vec{p}^{\prime}, s^{\prime}\right)\right\}=\delta^{3}\left(\vec{p}-\vec{p}^{\prime}\right) \delta_{s s^{\prime}}=\left\{d(\vec{p}, s), d^{*}\left(\vec{p}^{\prime}, s^{\prime}\right)\right\}, \\
\left\{c(\vec{p}, s), c\left(\vec{p}^{\prime}, s^{\prime}\right)\right\}=\left\{d(\vec{p}, s), d\left(\vec{p}^{\prime}, s^{\prime}\right)\right\}=\left\{c(\vec{p}, s), d\left(\vec{p}^{\prime}, s^{\prime}\right)\right\}=0 .
\end{array}
$$

The vacuum of Rarita-Schwinger fields is defined by the annihilation operators as

$$
c(\vec{p}, s)|0\rangle=d(\vec{p}, s)|0\rangle=0 .
$$

The evolution of free Rarita-Schwinger fields in proper time $\tau$ is generated by the free Hamiltonian given by

$$
H_{F}(\tau)=\sum_{s} \int d^{3} \vec{p} \omega_{\vec{p}}\left[c^{*}(\vec{p}, s) c(\vec{p}, s)+d^{*}(\vec{p}, s) d(\vec{p}, s)\right] \frac{d t}{d \tau} .
$$

The atom interacts with vacuum Rarita-Schwinger fields and we assume the interaction Hamiltonian to be

$$
H_{I}(\tau)=\mu R_{2}(\tau) \partial_{\alpha} \bar{\psi}^{\mu}(x(\tau)) \partial^{\alpha} \psi_{\mu}(x(\tau))
$$


where $\bar{\psi}^{\mu}(x(\tau))=\psi^{\mu^{\dagger}}(x(\tau)) \gamma^{0}, \mu$ is the coupling constant that is assumed to be small and $R_{2}(0)=\frac{1}{2} i\left[R_{-}(0)-R_{+}(0)\right]$ with $R_{+}(0)=|+\rangle\langle-|$ and $R_{-}(0)=|-\rangle\langle+|$ being the atomic raising and lowering operators respectively. These operators obey $\left[R_{3}, R_{ \pm}\right]= \pm R_{ \pm}$, and $\left[R_{+}, R_{-}\right]=2 R_{3}$. Similar to the case of Dirac fields[14], the interaction Hamiltonian here is also quadratic in the field operator. This type of nonlinear interaction differs remarkably from the linear atom-field couplings in the scalar and electromagnetic field cases [5-13] in the sense that it makes atomic transitions via both absorption and emission of Rarita-Schwinger particle-antiparticle pairs and inelastic scattering of a particle or antiparticle possible at the lowest order of perturbation, whereas in the linear coupling case, the quantum is singly absorbed or emitted and inelastic scattering occurs only at higher orders.

Now, with the total Hamiltonian of the system (atom+field) which is given by: $H(\tau)=$ $H_{A}(\tau)+H_{F}(\tau)+H_{I}(\tau)$, we can derive the Heisenberg equations of motion for the dynamical variables of the atom and the field, and their solutions can be separated into two parts: the free part (denoted by index "f") that is present even if no coupling between the atom and the field is assumed to exist and the source part (denoted by index "s") characterized by the coupling constant $\mu$, which is generated by the interaction. They are associated with vacuum fluctuations and radiation reaction, respectively. If we assume that the state of the system is $|0, b\rangle$, where 0 represents the vacuum state of the field and $b$ the state of the atom, and choose a symmetric ordering between atom and field variables [15, 16], then following the same procedure as that in Ref. [14], we find, to the order $\mu^{2}$, the mean rate of change of the atomic energy

$$
\left\langle\frac{d H_{A}(\tau)}{d \tau}\right\rangle=\left\langle\frac{d H_{A}(\tau)}{d \tau}\right\rangle_{v f}+\left\langle\frac{d H_{A}(\tau)}{d \tau}\right\rangle_{\text {cross }}
$$

where

$$
\begin{aligned}
\left\langle\frac{d H_{A}(\tau)}{d \tau}\right\rangle_{v f}= & \frac{1}{2} i \mu \omega_{0}\left\langle\partial_{\alpha} \bar{\psi}^{\mu f}(x(\tau)) \partial^{\alpha} \psi_{\mu}{ }^{f}(x(\tau))\left[R_{2}(\tau), R_{3}(\tau)\right]\right. \\
& \left.+\left[R_{2}(\tau), R_{3}(\tau)\right] \partial_{\alpha} \bar{\psi}^{\mu f}(x(\tau)) \partial^{\alpha} \psi_{\mu}{ }^{f}(x(\tau))\right\rangle \\
\left\langle\frac{d H_{A}(\tau)}{d \tau}\right\rangle_{\text {cross }}= & \frac{1}{2} i \mu \omega_{0}\left\langle\partial_{\alpha} \bar{\psi}^{\mu f}(x(\tau)) \partial^{\alpha} \psi_{\mu}{ }^{s}(x(\tau))\left[R_{2}(\tau), R_{3}(\tau)\right]\right. \\
& +\left[R_{2}(\tau), R_{3}(\tau)\right] \partial_{\alpha} \bar{\psi}^{\mu f}(x(\tau)) \partial^{\alpha} \psi_{\mu}{ }^{s}(x(\tau)) \\
& +\partial_{\alpha} \bar{\psi}^{\mu s}(x(\tau)) \partial^{\alpha} \psi_{\mu}{ }^{f}(x(\tau))\left[R_{2}(\tau), R_{3}(\tau)\right] \\
& \left.+\left[R_{2}(\tau), R_{3}(\tau)\right] \partial_{\alpha} \bar{\psi}^{\mu s}(x(\tau)) \partial^{\alpha} \psi_{\mu}{ }^{f}(x(\tau))\right\rangle .
\end{aligned}
$$

Notice that the cross-term contains both the free part and the source part and it is of the same order as the sole vacuum fluctuation term in our perturbative treatment correct to the order $\mu^{2}$. The cross-term dominates however over the sole radiation reaction term which is of order $\mu^{3}$ and which can thus be neglected. This term does not appear at the order $\mu^{2}$ in 
the cases of linear couplings [5-13]. The above equations can be further simplified to

$$
\begin{aligned}
\left\langle\frac{d H_{A}(\tau)}{d \tau}\right\rangle_{v f} & =2 i \mu^{2} \int_{\tau_{0}}^{\tau} d \tau^{\prime} C^{F}\left(x(\tau), x\left(\tau^{\prime}\right)\right) \frac{d}{d \tau} \chi^{A}\left(\tau, \tau^{\prime}\right), \\
\left\langle\frac{d H_{A}(\tau)}{d \tau}\right\rangle_{\text {cross }} & =2 i \mu^{2} \int_{\tau_{0}}^{\tau} d \tau^{\prime} \chi^{F}\left(x(\tau), x\left(\tau^{\prime}\right)\right) \frac{d}{d \tau} C^{A}\left(\tau, \tau^{\prime}\right) .
\end{aligned}
$$

Here $C^{F}\left(x(\tau), x\left(\tau^{\prime}\right)\right)$ and $\chi^{F}\left(x(\tau), x\left(\tau^{\prime}\right)\right)$ are the two statistical functions of the field defined respectively as

$$
C^{F}\left(x(\tau), x\left(\tau^{\prime}\right)\right)=C_{+}^{F}\left(x(\tau), x\left(\tau^{\prime}\right)\right)+C_{-}^{F}\left(x(\tau), x\left(\tau^{\prime}\right)\right)
$$

and

$$
\chi^{F}\left(x(\tau), x\left(\tau^{\prime}\right)\right)=\chi_{+}^{F}\left(x(\tau), x\left(\tau^{\prime}\right)\right)+\chi_{-}^{F}\left(x(\tau), x\left(\tau^{\prime}\right)\right)
$$

with

$$
\begin{aligned}
& C_{+}^{F}\left(x(\tau), x\left(\tau^{\prime}\right)\right)=\frac{1}{2}\left\langle 0\left|\partial_{\alpha} \bar{\psi}^{\mu f}(x(\tau)) \partial^{\alpha} \psi_{\mu}{ }^{f}(x(\tau)) \partial_{\beta}^{\prime} \bar{\psi}^{\nu f}\left(x\left(\tau^{\prime}\right)\right) \partial^{\prime \beta} \psi_{\nu}{ }^{f}\left(x\left(\tau^{\prime}\right)\right)\right| 0\right\rangle, \\
& C_{-}^{F}\left(x(\tau), x\left(\tau^{\prime}\right)\right)=\frac{1}{2}\left\langle 0\left|\partial_{\beta}^{\prime} \bar{\psi}^{\nu f}\left(x\left(\tau^{\prime}\right)\right) \partial^{\prime \beta} \psi_{\nu}{ }^{f}\left(x\left(\tau^{\prime}\right)\right) \partial_{\alpha} \bar{\psi}^{\mu f}(x(\tau)) \partial^{\alpha} \psi_{\mu}{ }^{f}(x(\tau))\right| 0\right\rangle,
\end{aligned}
$$

and

$$
\begin{aligned}
& \chi_{+}^{F}\left(x(\tau), x\left(\tau^{\prime}\right)\right)=-\frac{1}{2}\left\langle 0\left|\partial_{\alpha} \bar{\psi}^{\mu f}(x(\tau))\left[\partial_{\beta}^{\prime} \bar{\psi}^{\nu f}\left(x\left(\tau^{\prime}\right)\right) \partial^{\prime \beta} \psi_{\nu}^{f}\left(x\left(\tau^{\prime}\right)\right), \partial^{\alpha} \psi_{\mu}^{f}(x(\tau))\right]\right| 0\right\rangle, \\
& \chi_{-}^{F}\left(x(\tau), x\left(\tau^{\prime}\right)\right)=-\frac{1}{2}\left\langle 0\left|\left[\partial_{\beta}^{\prime} \bar{\psi}^{\nu f}\left(x\left(\tau^{\prime}\right)\right) \partial^{\prime \beta} \psi_{\nu}^{f}\left(x\left(\tau^{\prime}\right)\right), \partial_{\alpha} \bar{\psi}^{\mu f}(x(\tau))\right] \partial^{\alpha} \psi_{\mu}^{f}(x(\tau))\right| 0\right\rangle,
\end{aligned}
$$

while $C^{A}\left(\tau, \tau^{\prime}\right)$ and $\chi^{A}\left(\tau, \tau^{\prime}\right)$ are the two susceptibility functions of the atom

$$
\begin{aligned}
C^{A}\left(\tau, \tau^{\prime}\right) & =\frac{1}{2}\left\langle b\left|\left\{R_{2}^{f}(\tau), R_{2}^{f}\left(\tau^{\prime}\right)\right\}\right| b\right\rangle, \\
\chi^{A}\left(\tau, \tau^{\prime}\right) & =\frac{1}{2}\left\langle b\left|\left[R_{2}^{f}(\tau), R_{2}^{f}\left(\tau^{\prime}\right)\right]\right| b\right\rangle,
\end{aligned}
$$

which can be explicitly written as

$$
\begin{aligned}
& C^{A}\left(\tau, \tau^{\prime}\right)=\frac{1}{2} \sum_{d}\left|\left\langle b\left|R_{2}(0)\right| d\right\rangle\right|^{2}\left(e^{i \omega_{b d}\left(\tau-\tau^{\prime}\right)}+e^{-i \omega_{b d}\left(\tau-\tau^{\prime}\right)}\right), \\
& \chi^{A}\left(\tau, \tau^{\prime}\right)=\frac{1}{2} \sum_{d}\left|\left\langle b\left|R_{2}(0)\right| d\right\rangle\right|^{2}\left(e^{i \omega_{b d}\left(\tau-\tau^{\prime}\right)}-e^{-i \omega_{b d}\left(\tau-\tau^{\prime}\right)}\right) .
\end{aligned}
$$

The summation in the above two equations runs over the complete set of the states of the atom. 


\section{THE SPONTANEOUS EXCITATION IN VACUUM WITH RARITA- SCHWINGER FIELD FLUCTUATIONS}

Let us assume that the two-level atom be uniformly accelerated such that its trajectory is described by

$$
t(\tau)=\frac{1}{a} \sinh (a \tau), \quad x(\tau)=\frac{1}{a} \cosh (a \tau), \quad y(\tau)=z(\tau)=0,
$$

where $a$ is the proper acceleration. Now we begin to calculate the mean rate of change of the atomic energy of the uniformly accelerated atom using the formalism given in the preceding section. To do this, we need to compute the statistical functions of Rarita-Schwinger fields. To simplify our computation, we define a matrix related to the two-point function of RaritaSchwinger fields as follows:

$$
S_{\mu \nu}^{+}\left(x(\tau), x\left(\tau^{\prime}\right)\right)=\left\langle 0\left|\psi_{\mu}(x(\tau)) \bar{\psi}_{\nu}\left(x\left(\tau^{\prime}\right)\right)\right| 0\right\rangle .
$$

Similarly, we can also define another one $S_{\mu \nu}^{-}\left(x(\tau), x\left(\tau^{\prime}\right)\right)$ by:

$$
\left[S_{\mu \nu}^{-}\left(x(\tau), x\left(\tau^{\prime}\right)\right)\right]_{a b}=\left\langle 0\left|\left(\bar{\psi}_{\nu}\left(x\left(\tau^{\prime}\right)\right)\right)_{b}\left(\psi_{\mu}(x(\tau))\right)_{a}\right| 0\right\rangle
$$

These two matrices are positive and negative frequency Wightman functions of RaritaSchwinger fields, respectively. Substituting Eq. (66) into Eq. (29), and utilizing Eqs. (11) - (14), we obtain

$$
\begin{aligned}
S_{\mu \nu}^{+}\left(x(\tau), x\left(\tau^{\prime}\right)\right) & =\sum_{s= \pm \frac{3}{2}} \int \frac{d^{3} \vec{p}}{(2 \pi)^{3} 2 p_{0}} u_{\mu}(\vec{p}, s) \bar{u}_{\nu}(\vec{p}, s) e^{-i p\left(x-x^{\prime}\right)} \\
& =-\frac{i}{2} \bar{\delta}_{\nu \alpha}\left(\gamma_{\alpha} \not \partial \gamma_{\beta}\right) \bar{\delta}_{\beta \mu} G^{+}\left(x(\tau), x\left(\tau^{\prime}\right)\right)
\end{aligned}
$$

where

$$
\bar{\delta}_{\mu \nu}=\epsilon_{\mu}(\vec{p},+) \epsilon_{\nu}^{*}(\vec{p},+)+\epsilon_{\mu}(\vec{p},-) \epsilon_{\nu}^{*}(\vec{p},-)=\delta_{\mu \nu}-\left(\partial_{\mu} \bar{\partial}_{\nu}+\partial_{\nu} \bar{\partial}_{\mu}\right)(\partial \bar{\partial})^{-1}
$$

with $\bar{\partial}_{\mu}=\left(-\partial_{0}, \partial_{i}\right), \partial \bar{\partial}=\partial^{\alpha} \bar{\partial}_{\alpha}$. In the Coulomb-like gauge (5), there are no unphysical modes in the function above. Similarly, one can also show that

$$
S_{\mu \nu}^{-}\left(x(\tau), x\left(\tau^{\prime}\right)\right)=\frac{i}{2} \bar{\delta}_{\nu \alpha}\left(\gamma_{\alpha} \not \partial \gamma_{\beta}\right) \bar{\delta}_{\beta \mu} G^{-}\left(x(\tau), x\left(\tau^{\prime}\right)\right)
$$

Here $G^{+}\left(x(\tau), x\left(\tau^{\prime}\right)\right)$ and $G^{-}\left(x(\tau), x\left(\tau^{\prime}\right)\right)$ are positive and negative frequency Wightman functions of massless scalar fields in four dimensional Minkowski spacetime, which are given respectively by

$$
\begin{aligned}
G^{+}\left(x(\tau), x\left(\tau^{\prime}\right)\right) & =-\frac{1}{4 \pi^{2}} \frac{1}{(\triangle \tau-i \varepsilon)^{2}-\left|\vec{x}-\vec{x}^{\prime}\right|^{2}} \\
G^{-}\left(x(\tau), x\left(\tau^{\prime}\right)\right) & =-\frac{1}{4 \pi^{2}} \frac{1}{(\triangle \tau+i \varepsilon)^{2}-\left|\vec{x}^{\prime}-\vec{x}\right|^{2}}
\end{aligned}
$$


where $\triangle \tau=\tau-\tau^{\prime}$ and $\varepsilon$ is a real infinitesimal quantity. Exchanging $x(\tau)$ and $x\left(\tau^{\prime}\right)$ in $S_{\mu \nu}^{+}\left(x(\tau), x\left(\tau^{\prime}\right)\right)$ leads to

$$
S_{\mu \nu}^{+}\left(x\left(\tau^{\prime}\right), x(\tau)\right)=\frac{i}{2} \bar{\delta}_{\nu \alpha}\left(\gamma_{\alpha} \not \partial \gamma_{\beta}\right) \bar{\delta}_{\beta \mu} G^{+}\left(x\left(\tau^{\prime}\right), x(\tau)\right) .
$$

Thus, noticing that $G^{-}\left(x(\tau), x\left(\tau^{\prime}\right)\right)=G^{+}\left(x\left(\tau^{\prime}\right), x(\tau)\right)$, we arrive at the relation:

$$
S_{\mu \nu}^{-}\left(x(\tau), x\left(\tau^{\prime}\right)\right)=S_{\mu \nu}^{+}\left(x\left(\tau^{\prime}\right), x(\tau)\right) .
$$

With the Wightman functions of massless Rarita-Schwinger fields derived, now we can calculate the two statistical functions needed in our calculation of the mean rate of change of the atomic energy. Using Eqs. (29) and (30), we see that $C_{+}^{F}\left(x(\tau), x\left(\tau^{\prime}\right)\right)$ and $C_{-}^{F}\left(x(\tau), x\left(\tau^{\prime}\right)\right)$ can be written respectively as:

$$
\begin{aligned}
& C_{+}^{F}\left(x(\tau), x\left(\tau^{\prime}\right)\right)=\operatorname{Tr}\left[\partial^{\alpha} \partial^{\prime}{ }_{\beta} S_{\mu}^{\nu+}\left(x(\tau), x\left(\tau^{\prime}\right)\right) \partial_{\alpha} \partial^{\prime \beta} S_{\nu}^{\mu-}\left(x\left(\tau^{\prime}\right), x(\tau)\right)\right], \\
& C_{-}^{F}\left(x(\tau), x\left(\tau^{\prime}\right)\right)=\operatorname{Tr}\left[\partial_{\alpha} \partial^{\prime \beta} S_{\nu}^{\mu-}\left(x\left(\tau^{\prime}\right), x(\tau)\right) \partial^{\alpha} \partial^{\prime}{ }_{\beta} S_{\mu}^{\nu+}\left(x(\tau), x\left(\tau^{\prime}\right)\right)\right],
\end{aligned}
$$

and $\chi_{+}^{F}\left(x(\tau), x\left(\tau^{\prime}\right)\right)$ and $\chi_{-}^{F}\left(x(\tau), x\left(\tau^{\prime}\right)\right)$ respectively as:

$$
\begin{aligned}
\chi_{+}^{F}\left(x(\tau), x\left(\tau^{\prime}\right)\right)= & -\operatorname{Tr}\left[\partial_{\alpha} \partial^{\prime \beta} S_{\nu}^{\mu-}\left(x\left(\tau^{\prime}\right), x(\tau)\right) \partial_{\beta}^{\prime} \partial^{\alpha} S_{\mu}^{\nu-}\left(x(\tau), x\left(\tau^{\prime}\right)\right)\right] \\
& -\operatorname{Tr}\left[\partial_{\alpha} \partial^{\prime \beta} S_{\nu}^{\mu-}\left(x\left(\tau^{\prime}\right), x(\tau)\right) \partial^{\alpha} \partial_{\beta}^{\prime} S_{\mu}^{\nu+}\left(x(\tau), x\left(\tau^{\prime}\right)\right)\right], \\
\chi_{-}^{F}\left(x(\tau), x\left(\tau^{\prime}\right)\right)= & \operatorname{Tr}\left[\partial_{\beta}^{\prime} \partial^{\alpha} S_{\mu}^{\nu-}\left(x(\tau), x\left(\tau^{\prime}\right)\right) \partial_{\beta}^{\prime} \partial^{\alpha} S_{\nu}^{\mu+}\left(x\left(\tau^{\prime}\right), x(\tau)\right)\right] \\
& +\operatorname{Tr}\left[\partial_{\alpha} \partial^{\prime \beta} S_{\nu}^{\mu-}\left(x\left(\tau^{\prime}\right), x(\tau)\right) \partial_{\beta}^{\prime} \partial^{\alpha} S_{\mu}^{\nu-}\left(x(\tau), x\left(\tau^{\prime}\right)\right)\right],
\end{aligned}
$$

where $\operatorname{Tr}[\cdots]$ represents the trace of a matrix. Substituting Eqs. (31) and (33) into Eqs. (37)(40), and using Eqs. (34), (36) and the trajectory (28), we get the two statistical functions:

$$
\begin{aligned}
& C^{F}\left(x(\tau), x\left(\tau^{\prime}\right)\right)=-\frac{183 a^{10}}{32 \pi^{4}}\left[\frac{1}{\sinh ^{10}\left(\frac{a}{2} \Delta \tau-i \varepsilon\right)}+\frac{1}{\sinh ^{10}\left(\frac{a}{2} \Delta \tau+i \varepsilon\right)}\right], \\
& \chi^{F}\left(x(\tau), x\left(\tau^{\prime}\right)\right)=-\frac{183 a^{10}}{32 \pi^{4}}\left[\frac{1}{\sinh ^{10}\left(\frac{a}{2} \Delta \tau-i \varepsilon\right)}-\frac{1}{\sinh ^{10}\left(\frac{a}{2} \Delta \tau+i \varepsilon\right)}\right],
\end{aligned}
$$

Inserting Eqs. (41) and (27) into Eq. (20), extending the range of integration to infinity for sufficiently long proper times, and making use of the techniques of contour integration and residue theory, we obtain the contribution of vacuum fluctuations to the mean rate of change of the atomic energy:

$$
\begin{aligned}
\left\langle\frac{d H_{A}(\tau)}{d \tau}\right\rangle_{v f}=- & \frac{61 \mu^{2}}{1890 \pi^{3}} \sum_{\omega_{b}>\omega_{d}}\left|\left\langle b\left|R_{2}(0)\right| d\right\rangle\right|^{2} \omega_{b d}^{10} \\
& \times\left(1+30 \frac{a^{2}}{\omega_{b d}^{2}}+273 \frac{a^{4}}{\omega_{b d}^{4}}+820 \frac{a^{6}}{\omega_{b d}^{6}}+576 \frac{a^{8}}{\omega_{b d}^{8}}\right)\left(1+\frac{2}{e^{2 \pi \omega_{b d} / a}-1}\right) \\
& +\frac{61 \mu^{2}}{1890 \pi^{3}} \sum_{\omega_{b}<\omega_{d}}\left|\left\langle b\left|R_{2}(0)\right| d\right\rangle\right|^{2} \omega_{b d}^{10} \\
& \times\left(1+30 \frac{a^{2}}{\omega_{b d}^{2}}+273 \frac{a^{4}}{\omega_{b d}^{4}}+820 \frac{a^{6}}{\omega_{b d}^{6}}+576 \frac{a^{8}}{\omega_{b d}^{8}}\right)\left(1+\frac{2}{e^{2 \pi\left|\omega_{b d}\right| / a}-1}\right) .
\end{aligned}
$$


This reveals that the contributions of vacuum Rarita-Schwinger field fluctuations would increase the atomic energy when the atom is initially in its ground state ( because of the term $\left.\left(\omega_{b}<\omega_{d}\right)\right)$ and decrease it if otherwise (because of the term $\left(\omega_{b}>\omega_{d}\right)$ ). What distinguishes a uniformly accelerated atom in interaction with the Rarita-Schwinger field fluctuations from that in interaction with massless scalar field, electromagnetic field and Dirac field fluctuations is the appearance of extra terms which are proportional to $a^{6}$ and $a^{8}$.

Similarly, we can find, by plugging Eqs. (42) and (26) into Eq. (21), the contribution of the cross term to the mean rate of change of the atomic energy:

$$
\begin{aligned}
\left\langle\frac{d H_{A}(\tau)}{d \tau}\right\rangle_{\text {cross }}= & -\frac{61 \mu^{2}}{1890 \pi^{3}} \sum_{\omega_{b}>\omega_{d}}\left|\left\langle b\left|R_{2}(0)\right| d\right\rangle\right|^{2} \omega_{b d}^{10} \\
& \times\left(1+30 \frac{a^{2}}{\omega_{b d}^{2}}+273 \frac{a^{4}}{\omega_{b d}^{4}}+820 \frac{a^{6}}{\omega_{b d}^{6}}+576 \frac{a^{8}}{\omega_{b d}^{8}}\right) \\
& -\frac{61 \mu^{2}}{1890 \pi^{3}} \sum_{\omega_{b}<\omega_{d}}\left|\left\langle b\left|R_{2}(0)\right| d\right\rangle\right|^{2} \omega_{b d}^{10} \\
& \times\left(1+30 \frac{a^{2}}{\omega_{b d}^{2}}+273 \frac{a^{4}}{\omega_{b d}^{4}}+820 \frac{a^{6}}{\omega_{b d}^{6}}+576 \frac{a^{8}}{\omega_{b d}^{8}}\right) .
\end{aligned}
$$

This tells us that the contribution of the cross term can only decrease the atomic energy no matter what the initial state of the atom is. Adding up Eqs. (43) and (44), we obtain the total mean rate of change of the atomic energy

$$
\begin{aligned}
\left\langle\frac{d H_{A}(\tau)}{d \tau}\right\rangle_{t o t}=- & \frac{61 \mu^{2}}{945 \pi^{3}} \sum_{\omega_{b}>\omega_{d}}\left|\left\langle b\left|R_{2}(0)\right| d\right\rangle\right|^{2} \omega_{b d}^{10} \\
& \times\left(1+30 \frac{a^{2}}{\omega_{b d}^{2}}+273 \frac{a^{4}}{\omega_{b d}^{4}}+820 \frac{a^{6}}{\omega_{b d}^{6}}+576 \frac{a^{8}}{\omega_{b d}^{8}}\right)\left(1+\frac{1}{e^{2 \pi \omega_{b d} / a}-1}\right) \\
& +\frac{61 \mu^{2}}{945 \pi^{3}} \sum_{\omega_{b}<\omega_{d}}\left|\left\langle b\left|R_{2}(0)\right| d\right\rangle\right|^{2} \omega_{b d}^{10} \\
& \times\left(1+30 \frac{a^{2}}{\omega_{b d}^{2}}+273 \frac{a^{4}}{\omega_{b d}^{4}}+820 \frac{a^{6}}{\omega_{b d}^{6}}+576 \frac{a^{8}}{\omega_{b d}^{8}}\right) \frac{1}{e^{2 \pi\left|\omega_{b d}\right| / a}-1} .
\end{aligned}
$$

As can be seen, from the above expression, the uniformly accelerated atom in the ground state would spontaneously transition to an excited state in vacuum with fluctuating RaritaSchwinger fields. When $a \gg \omega_{b d}$, the terms proportional to $a^{6}$ and $a^{8}$ which distinguish the case of the Rarita-Schwinger fields from the cases of scalar, electromagnetic and Dirac fields become preponderant over other terms. The appearance of power terms in $a$ in the mean rate of change of atomic energy for accelerated atoms in interaction with vacuum electromagnetic, Dirac, as well as Rarita-Schwinger fields we are discussing in the present 
paper, which are absent in the case of a scalar field, shows that the equivalence between the acceleration and a thermal bath is lost in terms of the spontaneous excitation of atoms in these cases. However, the deviation from pure thermal behavior of the spontaneous excitation of the uniformly accelerated atom by no means implies the exact final thermal equilibrium is not reached. In fact, with the transition probabilities (excitation and emission) for the uniformly accelerated atom which can be found from Eq.(45), one can show, by the same argument as that in Ref [24], that exact thermal equilibrium will be established at the Unruh temperature. Nevertheless, different behaviors of the transition probabilities of the atoms do imply a clear difference between a thermal bath and the acceleration in terms of how atomic transitions occur and how the equilibrium is reached.

\section{SUMMARY}

We have calculated, after generalizing the DDC formalism to the case of an atom coupled nonlinearly to vacuum Rarita-Schwinger field fluctuations, the spontaneous excitation of a uniformly accelerated atom in interaction with fluctuating vacuum Rarita-Schwinger fields. Our result shows that such a uniformly accelerated atom in its ground state would spontaneously transition to an excited state in vacuum and the excitation rate contains, in addition to those associated with a pure thermal bath the Unruh temperature at $T=a / 2 \pi$, terms proportional to the acceleration to the power of six and eight which are absent in the cases of the scalar, electromagnetic and Dirac fields. Although the behavior of a uniformly accelerated atom deviates from that in a thermal bath in terms of the spontaneous excitation and emission due to the appearance of such power terms, the exact thermal equilibrium will still be established at the Unruh temperature $a / 2 \pi$. Nevertheless, different behaviors of the transition probabilities of the atoms do imply a clear distinguishability between a thermal bath and the acceleration in terms of how atomic transitions occur.

We must point out that the appearance in the excitation rate of terms of higher powers of acceleration compared to the case of Dirac fields [14], i.e., terms proportional to the sixth and eighth powers of acceleration, is actually a result of the derivative coupling nature of the interaction. The same thing happens in the scalar field case, where powers of acceleration will appear in the excitation rate of a detector when a monopole coupling is replaced by a derivative coupling [4, 10, 25, 27]. This is because of that the excitation rate depends on the derivatives of the Wightman function, which increase the order of the pole in the Sinh function. The higher the order of the pole, the higher the powers of acceleration in the excitation rate. It is also interesting to note that derivative coupling is not the sole reason for the increase in the order of the pole, in fact, any perturbative expansion in a small parameter of the Wightman function, even for the monopole coupling case is sufficient(see, for example, Refs. [28, 29]).

Finally, let us note that when one considers that the detector is switched on and off, then 
the choice of the form of the Wightman function Eq. (34) in the present paper corresponds to a particular choice of the regularizaion function which requires smooth $C^{\infty}$ switching

functions [30]. In this switching-on-and-off scenario, the switching functions considered by us here are actually Heavyside step functions which usually result in transient effects due to switching even for a stationary trajectory. The absence of such effects in the present paper is however a result of the fact that we assume the detector to be switched on for an infinite time interval which makes the extra transient effects vanish.

\section{Acknowledgments}

This work was supported in part by the NNSFC under Grants No. 11075083 and No. 11375092; the National 973 Program of China under Grant No. 2010CB832803; the PCSIRT under Grant No. IRT0964; the Hunan Provincial Natural Science Foundation under Grant No. 11JJ7001 and the SRFDP under Grant No. 20124306110001

[1] W.G. Unruh, Phys. Rev. D 14, 870 (1976).

[2] S. Takagi, Prog. Theor. Phys. Suppl. 88, 1 (1986).

[3] T. Padmanabhan, Phys. Rep. 406, 49 (2005).

[4] L. C. B. Crispino, A. Higuchi, and G. E. A. Matsas, Rev. Mod. Phys. 80, 787 (2008).

[5] J. Audretsch and R. Müller, Phys. Rev. A 50, 1755 (1994).

[6] J. Audretsch and R. Müller, Phys. Rev. A 52, 629 (1995).

[7] J. Audretsch, R. Müller and M. Holzmann, Class. Quant. Grav. 12, 2927 (1995).

[8] R. Passante, Phys. Rev. A 57, 1590 (1998).

[9] Z. Zhu, H. Yu and S. Lu, Phys. Rev. D 73, 107501 (2006).

[10] H. Yu and Z. Zhu, Phys. Rev. D 74, 044032 (2006).

[11] L. Rizzuto, Phys. Rev. A 76, 062114 (2007).

[12] L. Rizzuto and S. Spagnolo, Phys. Rev. A 79, 062110 (2009).

[13] Z. Zhu and H. Yu, Phys. Rev. A 82, 042108 (2010).

[14] W. Zhou and H. Yu, Phys. Rev. A 86, 033841 (2012).

[15] J. Dalibard, J. Dupont-Roc and C. Cohen-Tannoudji, J. Phys. (France) 43, 1617 (1982).

[16] J. Dalibard, J. Dupont-Roc and C. Cohen-Tannoudji, J. Phys. (France) 45, 637 (1984).

[17] W. Rarita and J. Schwinger, Phys. Rev. 60, 61(1941). 
[18] Y.A. Gol'fand and E.P. Likhtman, JETP Lett. 13 (1971) 323.

[19] D.V. Volkov and V.P. Akulov, JETP Lett. 16 (1972) 438.

[20] J. Wess and B. Zumino, Nucl. Phys. B70 (1974) 39.

[21] R. H. Dicke, Phys. Rev. 93, 99 (1954).

[22] P. Van Nieuwenhuizen, Phys. Rep. 68, 189 (1981)

[23] A. Das and D. Z. Freedman, Nucl. Phys. B114, 271(1976).

[24] B. Garbrecht and T. Prokopec, Class. Quantum. Grav. 21, 4993 (2004).

[25] K. J. Hinton, J. Phys. A 16, 1937 (1983).

[26] Y. Zhu, H. Yu and Z. Zhu Class. Quantum. Grav. 24, 95 (2007).

[27] E. Martin-Martinez and J. Louko, arXiv:1404.5621

[28] J.G. Russo and P.K. Townsend J.Phys.Conf.Ser.222, 012040 (2010).

[29] S. Kolekar and T. Padmanabhan, Phys. Rev. D 86, 104057 (2012).

[30] A. Satz, Class. Quantum. Grav. 24, 1719 (2007). 